\title{
Magnetic Sphincter Augmentation In Patients With Paraesophageal Hernia Vs. Large Sliding Hiatal Hernia: Comparison of Clinical Outcome And Complexity Of The Surgery
}

\section{Katrin Schwameis}

Allegheny Health Network

Shahin Ayazi ( $\square$ shahin.ayazi@gmail.com )

Allegheny Health Network

\section{Ping Zheng}

Allegheny Health Network

Andrew Grubic

Allegheny Health Network

Ali Zaidi

Allegheny Health Network

Adam Alleyne

Allegheny Health Network

Blair Jobe

Allegheny Health Network

\section{Research Article}

Keywords: paraesophageal hernia, sliding hiatal hernia, magnetic sphincter augmentation (MSA), gastroesophageal reflux disease (GERD)

Posted Date: May 13th, 2021

DOI: https://doi.org/10.21203/rs.3.rs-506952/v1

License: (c) (i) This work is licensed under a Creative Commons Attribution 4.0 International License. Read Full License

Version of Record: A version of this preprint was published at Journal of the American College of Surgeons on October 1st, 2020. See the published version at https://doi.org/10.1016/j.jamcollsurg.2020.07.052. 


\section{Abstract}

Introduction: Recent studies encourage the use of magnetic sphincter augmentation (MSA) in patients with large sliding hiatal hernia (LHH). However, the utility of MSA in patients with paraesophageal hernia (PEH) is poorly studied. The aim of this study is to compare the outcome and complexity of surgery in patients with $\mathrm{PEH}$ to those with $\mathrm{LHH}$.

Methods: A total of 37 ( $65 \%$ female) patients underwent PEH repair with MSA in our institution between 2013 and 2019. A group of 37, age/sex matched patients with LHH $(\geq 4 \mathrm{~cm})$ who underwent MSA formed the control group. The clinical outcome and the complexity of the surgery were then compared between groups.

Results: At a median follow-up of 25.3 (17-35) months, there was significant improvement in the GERDHRQL total-scores for PEH (18 vs. $3, p<0.001)$ and LHH (26 vs. $4, p<0.0001)$ patients when compared to preoperative values. The $\mathrm{PEH}$ group was similar to $\mathrm{LHH}$ group in regard to $\mathrm{pH}$-normalization $(71 \% \mathrm{vs}$. $64 \%, p=0.76)$ and freedom from PPI $(94.4 \%$ vs. $91.9 \%, p=1.00)$. Small asymptomatic hernia recurrence was seen in $19 \%$ and $18 \%$ of $\mathrm{LHH}$ and PEH patients, respectively $(p=0.546)$. One patient $(3 \%)$ required surgical intervention for symptomatic recurrence in each group. The rate of dysphagia and need for dilation were similar between the groups.

No significant differences in operative time, blood loss, and hospital stay were detected between patients with $\mathrm{PEH}$ and those with $\mathrm{LHH}$. However, there was a trend toward higher necessity for additional operative maneuvers $(40.5 \%$ vs $13.5 \%, p=00.17)$ and longer hospital stay in $\mathrm{PEH}$.

Conclusion: Despite the inherent differences between $\mathrm{LHH}$ and PEH, repair of hernia and MSA results in high rate of favorable outcome and low rate of recurrence in both groups. GERD symptom control, freedom from PPI and improvement in quality of life are comparable. However, the greater need for additional surgical maneuvers and longer hospital stay reflect the greater complexity of procedures for repair of PEH with MSA.

\section{Introduction}

Magnetic sphincter augmentation is increasingly utilized as an effective alternative to laparoscopic Nissen fundoplication in the treatment of patients with gastroesophageal reflux disease (GERD). ${ }^{1-3}$ Multiple studies have shown that these two anti-reflux procedures result in comparable postoperative GERD symptom control, rates of freedom from PPI and $\mathrm{pH}$ normalization. ${ }^{1,4,5} \mathrm{MSA}$ is a less invasive and more physiologic surgical option, it preserves the ability of patient to belch and has a lower rate of gas bloating. ${ }^{6}$ Recent studies have encouraged use of MSA in patients with large sliding hiatal hernias. ${ }^{7,8}$ Clinical outcomes after repair of hiatal hernia and MSA in patients with hernias $\geq 3 \mathrm{~cm}$ were comparable or superior to those of patients with small or no hiatal hernia indicating that hernia size has no significant effect on the clinical efficacy of MSA. ${ }^{7,8}$ Short- and intermediate-term recurrence rates were low and 
mostly presented as small, asymptomatic sliding hernias. The titanium beads of the Linx ${ }^{\circledR}$ device can induce an inflammatory host reaction in surrounding tissues resulting in the formation of a fibrotic encapsulation. Formation of this "circumferential biological mesh" can reinforce the crural repair and previous studies have proposed it as an explanation for the low recurrence rate after MSA. 7, 9, 10

Despite the inherent differences in anatomy and necessity for specific surgical maneuvers, both, the repair of paraesophageal hernias (PEH) and large sliding hernias can prove challenging and technically complex. There is paucity of data on efficacy of MSA in patients with PEH. Paraesophageal hernias, accounts for $<5 \%$ of hiatal hernias and are associated with the potential for severe complications such as bleeding, volvulus, incarceration and gastrointestinal perforation. ${ }^{11,12}$ Surgical repair of PEH represents a technically challenging procedure with the main goals of tension-free restoration of the esophageal length and crural reapproximation. The management strategies of PEH have considerably evolved over the last decades, but there is no consensus on the optimal surgical approach and details of surgical techniques such as use of mesh reinforcement and choice of antireflux procedure. ${ }^{11}$ The modern laparoscopic PEH repair is associated with an objective recurrence rate of over $50 \%{ }^{13} \mathrm{~A}$ variety of technical modifications and maneuvers has been proposed to minimize tension on the repair and subsequently prevent hernia recurrence.

Based on encouraging surgical outcomes and low recurrence rates in patients with large sliding hernias, we sought to evaluate the efficacy of magnetic sphincter augmentation in patients with paraesophageal hernias. The aim of this study was to assess and compare clinical outcomes and the surgical complexity of magnetic sphincter augmentation and hiatal hernia repair in patients with paraesophageal hernia and those with large sliding hernia.

\section{Methods}

\section{Study design and study population}

This retrospective study reviewed all patients with paraesophageal hernia (PEH) that underwent magnetic sphincter augmentation (MSA) and hiatal hernia repair between 01/2012 and 10/2019 at Allegheny Health Network hospitals (Pittsburgh, PA). Patients with prior foregut surgeries were excluded from this study. A total of 37 patients with PEH were included in this study (Figure 1). Control group consisted of age and sex matched group of patients with large sliding hernia who underwent MSA and hiatal hernia repair during the same time period. "Large sliding hernia" was defined as type I hiatal hernia $\geq 4 \mathrm{~cm}$ in size on esophagram and/or endoscopy (Figure 2). A detailed chart review of baseline characteristics and postoperative clinical outcomes, GERD specific quality of life and PPI use after MSA was performed. All surgical reports were reviewed, and the complexity of a procedure was evaluated based on the following parameters: operative time $(\mathrm{min})$, intraoperative blood loss $(\mathrm{ml})$, length of hospital stay as well as number and type of additional surgical maneuvers performed. The latter included the performance of anterior crural stitches, vagal nerve or esophagogastric (EGJ) fat pad mobilization, central tendon release, extensive mediastinal dissection, Collis gastroplasty and reinforcement of crural repair with mesh. 
Clinical outcomes and surgical complexity of the procedures were then compared between patients with paraesophageal hernia and those with large sliding hernia. This study was evaluated and approved by the Institutional Review Board of the Allegheny Health Network. All methods were carried out in accordance with relevant guidelines and regulations. Informed consent was obtained from all study participants.

\section{Preoperative Foregut Evaluation}

All patients underwent comprehensive foregut evaluation prior to surgery. The work up consisted of a detailed clinical evaluation, upper GI endoscopy, videoesophagram and esophageal functioning testing. Esophageal motility was assessed using high-resolution impedance manometry (HRIM) technology (Medtronics, Shoreview, MN, USA), and manometric findings were reported in accordance with the Chicago classification v3.0. Once off proton pump inhibitors for 10 days, patients either underwent ambulatory wireless 48-hour BRAVO pH monitoring (Medtronics, Shoreview, MN, USA) or multichannel intraluminal (MII)-impedance pH testing (Sandhill Scientific Inc., Highlands Ranch, CO). Patients were instructed to precisely document their food and fluid intakes in a diet diary. A DeMeester score $>14.7$ was considered abnormal acid exposure of the distal esophagus ${ }^{14}$. Impedance $\mathrm{pH}$ testing was mostly performed in patients with atypical reflux symptoms using previously described criteria. ${ }^{15}$ Additionally, a symptom correlation analysis was performed.

\section{Magnetic Sphincter Augmentation and Hiatal Hernia Repair}

The LINX® reflux management system (Ethicon, Johnson \& Johnson; Shoreview, MN), utilized for magnetic sphincter augmentation, consists of interlinked magnetic titanium beads and features a Roman Arch design assuring non-compressing device closure. ${ }^{16}$ Its dynamic design ensures that the esophageal range of motion is not limited.

All procedures were performed laparoscopically by an experienced foregut surgeon using standardized surgical techniques as described previously. ${ }^{17-19}$ The operation consisted of the following 4 steps: reduction of the hernia sac, complete posterior mediastinal esophageal mobilization with restoration of intraabdominal esophageal length ( $\geq 3$ centimeters), interrupted posterior crural closure and device placement at the level of the esophagogastric junction. It was ensured that the posterior vagal nerve trunk was located outside of the MSA device. Intraoperative esophagogastroscopy was performed in order to assist in identifying the anatomic esophagogastric junction and to assess device position. In order to achieve tension-free hernia repair in more complex cases, the performance of extensive mediastinal dissection or adjunct surgical procedures such as vagal nerve or esophagogastric junction (EGJ) fat pad mobilization, central tendon release, diaphragmatic relaxing incisions, anterior crural stitches and mesh augmentation was required (Figure 3).

A solid LINX-diet was started on the day of surgery to avoid development of dysphagia secondary to forming scar tissue surrounding the device. Patients were encouraged to eat small portions of solid food every hour while awake for the first two months after surgery. Patients were discharged home when they 
were tolerating a diet with no difficulty and were free of symptom of nausea. Postoperative follow-up visits were scheduled at 2 weeks, 6 weeks, 6 months and then yearly after surgery.

\section{Follow-up after Magnetic Sphincter augmentation}

During postoperative clinic visits at 2 weeks, 6 weeks, 6 months and then annually, gastrointestinal symptoms and use of proton pump inhibitor (PPI) were assessed. Patients were also asked to complete standardized questionnaires including the GERD-Health-related-Quality-of-Life (GERD-HRQL). ${ }^{20-22}$ The frequency and severity of persistent postoperative dysphagia was reported based on the GERD HRQL "difficulty swallowing" item and significant dysphagia was defined as a score $>3$. Patients with a followup $\geq 12$ months were scheduled for esophagogastroduodenoscopy and postoperative esophageal function testing.

\section{Statistical Analysis}

Statistical analysis was performed using SPSS ${ }^{\circledR}$ statistics 20.0 (IBM, Armonk, NY). Data are presented using median and interquartile range (IQR) or mean [range and/or standard deviation (SD)]. Statistical analysis appropriate for non-parametric data was used. Categorical variables were analyzed using the Fisher's exact test and continuous data were analyzed using the Wilcoxon signed rank test as appropriate. Statistical significance was defined as a $p$-value $<0.05$.

\section{Results}

The paraesophageal hernia (PEH) group consisted of 37 patients (65\% females) with a median age of 63 years (35-82) who underwent repair of hernia and magnetic sphincter augmentation. The age- and sexmatched control group consisted of 37 patients with a large sliding hernia (LHH). Patients with PEH had a median size of $5 \mathrm{~cm}$ while the median size of hernia in patients with LHH was $4.5 \mathrm{~cm}(p=0.560)$. Patients with LHH had higher rate of esophagitis and higher median GERD-HRQL total scores when compared to those with $\mathrm{PEH}$. The remaining baseline characteristics were comparable between the two groups (Table 1). 
Table 1

Demographics and baseline characteristics of patients with paraesophageal hernia (PEH) and large sliding hernia $(\mathrm{LHH})$ prior to magnetic sphincter augmentation.

\begin{tabular}{|llll|}
\hline & PEH & LHH & p-value \\
& $\mathbf{n = 3 7}$ & $\mathbf{n = 3 7}$ & \\
\hline Sex (Females) & $24(65)$ & $24(65)$ & 1.000 \\
\hline Age (Median, Range) & $63(35-82)$ & $64(39-86)$ & 0.642 \\
\hline Median BMI (IQR) & $29.4(\mathrm{IQR}, 25.9-32.2)$ & $29.2(\mathrm{IQR}, 26.7-31.8)$ & 0.974 \\
\hline Median hernia size (cm) & $5(\mathrm{IQR}, 4-5.8)$ & $4.5(\mathrm{IQR}, 4.3-4.8)$ & 0.560 \\
\hline Esophagitis present (\%) & $15(40.5 \%)$ & $19(51.4)$ & 0.484 \\
\hline DeMeester score (Median, IQR) & $37.7(\mathrm{IQR}, 22.1-52.6)$ & $39.7(\mathrm{IQR}, 22-53.5)$ & 0.699 \\
\hline GERD HRQL total score (Median, IQR) & $18(\mathrm{IQR}, 8-25)$ & $26(\mathrm{IQR}, 16-33)$ & 0.021 \\
\hline
\end{tabular}

The most frequently used device sizes were 14 and 15 . The median operative time was comparable between the two groups (PEH: $108 \mathrm{~min}$ vs. LHH: $93 \mathrm{~min}, p=0.443$ ) and blood loss was minimal in all patients. Table 2 shows a comparison of the surgical complexity of performed procedures between the two groups. While there were no significant differences in the number of specific surgical maneuvers performed and length of hospital stay, a trend towards later discharge from hospital and higher necessity for any additional operative maneuvers such as extensive mediastinal dissection and vagal nerve or EGJ fat pad mobilization was detected for patients with paraesophageal hernia. One patient (2.7\%) with PEH required mesh reinforcement using (FlexHD ${ }^{\circledR}$ mesh) and two patients (5.4\%) with PEH required diaphragm relaxing incisions. No patient in this series underwent Collis gastroplasty. 
Table 2

Surgical complexity and length of hospital stay based on hernia type

\begin{tabular}{|llll|}
\hline & $\begin{array}{l}\text { PEH } \\
(\mathbf{n = 3 7 )}\end{array}$ & $\begin{array}{l}\text { LHH } \\
(\mathbf{n = 3 7 )}\end{array}$ & -value \\
\hline Additional surgical maneuver(s) performed: & $15(40.5 \%)$ & $5(13.5 \%)$ & 0.017 \\
\hline Anterior crural suture(s) & $5(13.5)$ & $1(2.7)$ & 0.199 \\
\hline Vagal nerve or GEJ fat pad mobilization & $5(13.5)$ & $0(0)$ & 0.054 \\
\hline Central tendon release & $1(2.7)$ & $0(0)$ & 1.000 \\
\hline Need for extensive mediastinal dissection & $10(27 \%)$ & $4(11 \%)$ & 0.136 \\
\hline Need for Collis gastroplasty & 0 & 0 & $\mathrm{n} / \mathrm{a}$ \\
\hline Median (IQR) OR time (min) & $108(70-133)$ & $93(76-128)$ & 0.443 \\
\hline Median (IQR) blood loss (ml) & $5(5-10)$ & $5(5-5)$ & 0.387 \\
\hline Discharge home on the same day & $12 / 21(57 \%)$ & $26 / 32(81 \%)$ & 0.07 \\
\hline
\end{tabular}

At a median follow up of 25 months (IQR: 17-35), GERD-HRQL was significantly improved in all patients $(p<0.001)$. Postoperative clinical outcomes are presented in Table 3. Postoperative $\mathrm{pH}$ monitoring was performed in $65 \%(n=24)$ of PEH patients and $68 \%(n=25)$ of LHH patients. Rates of postoperative $\mathrm{pH}$ normalization were comparable between the groups (PEH: $71 \%$ vs. $\mathrm{LHH}: 64 \%, p=0.762$ ). There was significant decrease in median postoperative DeMeester score in PEH group (38 vs. $5, p<0.001)$ and LHH group (40 vs. $11, p<0.001$ ). Freedom from PPI was achieved in over $90 \%$ of patients in both groups.

Table 3

Clinical outcomes after MSA and hiatal hernia repair in patients with paraesophageal hernia and those with large sliding hernia.

\begin{tabular}{|llll|}
\hline & PEH & LHH & -value \\
\hline Median follow up time (months) & $\mathbf{n = 3 7 ( 5 0 \% )}$ & $\mathbf{n = 3 7 ( 5 0 \% )}$ & \\
\hline GERD HRQL Total Score: & $24.8(17-35)$ & $24.4(17-35)$ & 0.92 \\
Postop vs. Preop & 18 vs. 3 (p<0.001) & 26 vs $4(p<0.001)$ & $n / a$ \\
\hline pH normalization & & & \\
\hline Freedom from PPI & $71 \%$ & $64 \%$ & 0.76 \\
\hline Postop. dysphagia & $94 \%$ & $92 \%$ & 1.00 \\
\hline Small asymptomatic recurrence & $30 \%$ & $27 \%$ & 1.00 \\
\hline Recurrent hernia requiring revisional surgery & $3 \%$ & $19 \%$ & 0.546 \\
\hline
\end{tabular}


Further, rates of postoperative dysphagia were similar in both groups (PEH: $30 \%$ vs. LHH: $27 \%, p=1.000$ ). Endoscopic dilation was performed in $8 \mathrm{PEH}$ patients $(22 \%)$ and $12 \mathrm{LHH}$ patients $(32 \%),(p=0.433)$. A small asymptomatic hiatal hernia recurrence was detected endoscopically in $18 \%$ of PEH and $19 \%$ of LHH patients, $(p=0.546)$. Three percent of patients in each group underwent revisional surgery for symptomatic hernia recurrence (Fig. 4).

\section{Discussion}

Magnetic sphincter augmentation is increasingly utilized as alternative to laparoscopic fundoplication in the treatment of patients with gastroesophageal reflux disease. ${ }^{23}$ Sliding hiatal hernias are still much more common than paraesophageal hernias, but the prevalence of the latter seems to be increasing in the United States. ${ }^{24}$ In patients with type I hiatal hernias the esophagogastric junction has migrated above the level of the diaphragm whereas in those with paraesophageal hernias, there is relative preservation of posterolateral phrenoesophageal attachments around the EGJ. ${ }^{25}$ The inherent differences of paraesophageal and large siding hernias result in varying disease manifestation, necessity for specific surgical maneuvers and forms of postoperative failure. Surgical repair of type I hiatal hernias is indicated in case of symptomatic reflux disease. In contrast, surgical repair of symptomatic paraesophageal hernias is indicated to reduce the risk for severe complications which is estimated to be $1 \%$ per year. ${ }^{26}$ The performance of an antireflux procedure is an essential part of any PEH repair as it reduces the risk of hernia recurrence and eliminates gastroesophageal reflux. ${ }^{27}$

In this study, we compared the clinical outcomes and surgical complexity of magnetic sphincter augmentation and hiatal hernia repair in patients with paraesophageal hernia to those with large sliding hernia. An important finding of our study is that magnetic sphincter augmentation and hiatal hernia repair results in favorable outcomes in patients with PEH and those with LHH. Quality of life improved significantly in all patients after MSA and rates of postoperative $\mathrm{pH}$ normalization and freedom from PPI were comparable between PEH and LHH patients. While encouraging outcomes of magnetic sphincter augmentation in patients with larger sliding hernias have been reported previously $7,8,28$, the present study is the first to selectively evaluate the efficacy of MSA in patients with PEH and compare it to a matched group of patients with LHH. Furthermore, postoperative reflux control of PEH patients treated with MSA in our cohort was comparable to published outcomes following laparoscopic fundoplication. ${ }^{29}$ These findings indicate that MSA represents an effective alternative to laparoscopic fundoplication in PEH patients. The choice of antireflux procedure is partially based on esophageal body motility function. Negative experiences with historic circumferential devices placed around the GI tract such as the Angelchik device raised concerns regarding the effect of the Linx device on esophageal peristalsis. However, early data suggest that while MSA leads to increased esophageal outflow resistance esophageal motility remains unaltered due to a compensatory increase in contractile vigor. ${ }^{30}$

In this series, low recurrence rates of $<20 \%$ in both hernia groups were detected at an intermediate term follow up of 2 years. Similar to other MSA studies, the majority of recurrences consisted of asymptomatic 
small sliding hernias without device dislocation ${ }^{8}$. Revisional surgery is rarely indicated in patients with recurrence and is relatively uncomplicated compared to repair of a herniated fundoplication which requires dissection of the wrap from the hiatus and esophagus. ${ }^{8}$ While long-term results have to be awaited, our recurrence rates of $<20 \%$ following MSA compare favorably to published recurrence rates of over $50 \%$ after laparoscopic $\mathrm{PEH}$ repair at a 5 year follow up. ${ }^{13}$ It has to be emphasized that recurrences observed in patients with MSA mostly consisted of small, sliding hernias which rarely require revision.

The results of this study highlight greater technical complexity of the repair of a paraesophageal hernias compared to large sliding hernias. More adjunct surgical maneuvers were required for the repair of paraesophageal hernias. A trend toward more need for extensive mediastinal dissection was also detected in patients with PEH. Furthermore, a trend toward longer operative times with increasing hernia size in LHH patients was detected (median OR time: hernia size $\leq 5 \mathrm{~cm}, 88 \mathrm{~min}$ (IQR, 74-94) vs. hernia size $>5 \mathrm{~cm}, 118 \mathrm{~min}$ (IQR, 68-143); $p=0.545)$.

Patients with PEH were more likely to require overnight stay after surgery. This is likely reflective of presence of other comorbidities in this group. The two groups were matched in regard to age, eliminating older age as an explanation for longer hospital stay in PEH group. Previous studies have shown higher rate of cardiopulmonary comorbidities from chronic, larger volume compressive effects of paraesophageal herniation. ${ }^{31}$

The overall operative time did not differ significantly between the two hernia groups, however inherent differences between these two types of hernia led to allocation of surgical time to different steps of surgery in each group. Patients with LHH had a higher rate of preoperative esophagitis. This indicates a more severe reflux disease in these patients compared to those with PEH. Longstanding exposure of distal esophagus to irritant acidic gastric juice in patients with LHH may result in transmural esophageal wall inflammation. This may then result in creating more chronic inflammation and adhesions around the EGJ and distal esophagus, making the dissection of these area more challenging and time consuming in patients with $\mathrm{LHH}$. A recent study demonstrated that MSA leads to significant clinical improvement across the GERD severity spectrum. ${ }^{32}$ Contrary, successful surgical repair of paraesophageal hernia require complete reduction of hernia sac, this is usually a lengthy step of surgery that requires a more extensive mediastinal dissection often to the level of inferior pulmonary vein.

Laparoscopic PEH repair is proven to be challenging and technically demanding. ${ }^{27}$ A successful repair requires a tension-free hernia repair with minimizing both, axial and radial tensions. ${ }^{33}$ Several adjunct procedures and technical modifications have been described in the literature to achieve a tension-free repair. Axial tension can be reduced by performing extensive mediastinal dissection, vagal nerve or EGJ fat pad mobilization and Collis gastroplasty. The wedge-fundectomy Collis gastroplasty has been established as simple yet effective tension-reducing technique in patients with shortened esophagus of intraabdominal length $<3 \mathrm{~cm} .{ }^{34}$ To ensure an adequate diameter of the "neoesophagus", the esophageal lengthening procedure is performed over a bougie. ${ }^{35}$ Previous studies have shown that the performance 
of a Collis gastroplasty in patients with shortened esophagus is safe and effective while reducing recurrence rates. ${ }^{34,36}$ However, as reflected by our results, an extensive mediastinal dissection usually allows mobilization of adequate length of intraabdominal esophagus, this eliminated the need for Collis gastroplasty in our practice.

Another challenge in repair of $\mathrm{PEH}$ is crural reapproximation. Radial tension, particularly in patients with wide splayed crura, is a major culprit for recurrence after laparoscopic $\mathrm{PEH}$ repair. Adjunct tensionminimizing techniques include crural relaxing incisions, central tendon release and mesh reinforcement of the crural repair. Critical assessment of the hiatal tension after suture cruroplasty should guide the choice of one or more techniques. Mesh reinforcement of laparoscopic PEH repair is controversial and it plays a subordinate role in our practice. Despite reduced recurrence rates after reinforcement with permanent, synthetic mesh, associated complications such as erosion, mesh infection and increased rates of postoperative dysphagia discouraged its application. ${ }^{37} \mathrm{~A}$ safer alternative is the use of an absorbable mesh. However, despite promising early results, long term outcomes did not demonstrate significant differences in recurrence rates between $\mathrm{PEH}$ patients that were treated with and without mesh reinforcement. ${ }^{13,38}$ Recently, encouraging outcomes of laparoscopic $\mathrm{PEH}$ repair with the use of newer biosynthetic absorbable mesh (Poly-4-hydroxybutyrate; Phasix-St-mesh) has been reported. ${ }^{24}$ The combination of mesh reinforcement and other tension-reducing techniques in that study resulted in a low rate of hernia recurrence and no mesh-related complications at short term follow up. However, selective mesh reinforcement is unlikely to overcome extensive hiatal tension. ${ }^{27}$ Thus, the use of crural relaxing incisions on the right side, or less commonly left side, can prove beneficial. Using the harmonic scalpel, a full-thickness incision of the right crus parallel to the inferior vena cava (IVC) is recommended. A $3 \mathrm{~mm}$ cuff of tissue is spared along the IVC which is later used for fixation of a PTFE patch which covers the diaphragmatic defect. ${ }^{39}$

Paraesophageal hernias are reported to occur more commonly in females of older age. Similar gender and age distribution among the two groups in this series is the result of the matched study design. Outcomes of PEH patients were compared to a sex and age matched control group of large sliding hernias to eliminate the confounding role of these factors and add to the strength of our study. However, we acknowledge that the $\mathrm{PEH}$ patient population of this study which included only those selected to undergo MSA might not be a comprehensive representative for the overall PEH patient population. Other limitations of this study include its retrospective nature and the relatively small samples sizes of the groups. Further multi-center studies with a larger patient population are necessary to evaluate long-term outcomes and recurrence rates after MSA and hiatal hernia repair in patients with paraesophageal hernia.

\section{Conclusions}

Despite the inherent differences between $\mathrm{LHH}$ and $\mathrm{PEH}$, magnetic sphincter augmentation with hiatal hernia repair results in high rate of favorable outcome and low rate of recurrence in both, patients with paraesophageal hernia and those with large sliding hernia. GERD symptom control, freedom from PPI 
and improvement in quality of life are comparable between these groups. However, the higher need for additional surgical maneuvers and longer median hospital stay reflect the greater complexity of procedures for repair of $\mathrm{PEH}$ with magnetic sphincter augmentation.

\section{Declarations}

\section{Acknowldegment:}

$\mathrm{N} / \mathrm{A}$

\section{Author Disclosure:}

Drs. Jobe and Ayazi receive consulting fee from Johnson and Johnson. Drs. Schwameis, Zheng, Grubic, Zaidi and Mrs. Myers and Mr. Alleyne have no conflicts of interest or financial ties to disclose.

\section{Funding:}

This research received no specific grant or funding from any agency.

\section{References}

1. Reynolds JL, Zehetner J, Wu P, et al. Laparoscopic Magnetic Sphincter Augmentation vs Laparoscopic Nissen Fundoplication: A Matched-Pair Analysis of 100 Patients. J Am Coll Surg 2015;221:123-8.

2. Warren HF, Reynolds JL, Lipham JC, et al. Multi-institutional outcomes using magnetic sphincter augmentation versus Nissen fundoplication for chronic gastroesophageal reflux disease. Surg Endosc 2016;30:3289-96.

3. Ayazi S, Zheng P, Zaidi AH, et al. Clinical Outcomes and Predictors of Favorable Result after Laparoscopic Magnetic Sphincter Augmentation: Single-Institution Experience with More than 500 Patients. J Am Coll Surg 2020;230:733-743.

4. Bonavina L, Horbach T, Schoppmann SF, et al. Three-year clinical experience with magnetic sphincter augmentation and laparoscopic fundoplication. Surg Endosc 2020.

5. Saino G, Bonavina L, Lipham JC, et al. Magnetic Sphincter Augmentation for Gastroesophageal Reflux at 5 Years: Final Results of a Pilot Study Show Long-Term Acid Reduction and Symptom Improvement. J Laparoendosc Adv Surg Tech A 2015;25:787-92.

6. Ganz RA, Gostout CJ, Grudem J, et al. Use of a magnetic sphincter for the treatment of GERD: a feasibility study. Gastrointest Endosc 2008;67:287-94.

7. Rona KA, Reynolds J, Schwameis K, et al. Efficacy of magnetic sphincter augmentation in patients with large hiatal hernias. Surg Endosc 2017;31:2096-2102.

8. Buckley FP, 3rd, Bell RCW, Freeman K, et al. Favorable results from a prospective evaluation of 200 patients with large hiatal hernias undergoing LINX magnetic sphincter augmentation. Surg Endosc 2017. 
9. Woodward ER, Thomas HF, McAlhany JC. Comparison of crural repair and Nissen fundoplication in the treatment of esophageal hiatus hernia with peptic esophagitis. Ann Surg 1971;173:782-92.

10. Louie BE, Kapur S, Blitz M, et al. Length and pressure of the reconstructed lower esophageal sphincter is determined by both crural closure and Nissen fundoplication. J Gastrointest Surg 2013;17:236-43.

11. Arafat FO, Teitelbaum EN, Hungness ES. Modern treatment of paraesophageal hernia: preoperative evaluation and technique for laparoscopic repair. Surg Laparosc Endosc Percutan Tech 2012;22:297303.

12. Alicuben ET, Worrell SG, DeMeester SR. Impact of crural relaxing incisions, Collis gastroplasty, and non-cross-linked human dermal mesh crural reinforcement on early hiatal hernia recurrence rates. $J$ Am Coll Surg 2014;219:988-92.

13. Oelschlager BK, Pellegrini CA, Hunter JG, et al. Biologic prosthesis to prevent recurrence after laparoscopic paraesophageal hernia repair: long-term follow-up from a multicenter, prospective, randomized trial. J Am Coll Surg 2011;213:461-8.

14. Ayazi S, Lipham JC, Portale G, et al. Bravo catheter-free pH monitoring: normal values, concordance, optimal diagnostic thresholds, and accuracy. Clin Gastroenterol Hepatol 2009;7:60-7.

15. Hoppo T, Sanz AF, Nason KS, et al. How much pharyngeal exposure is "normal"? Normative data for laryngopharyngeal reflux events using hypopharyngeal multichannel intraluminal impedance (HMII). J Gastrointest Surg 2012;16:16-24; discussion 24-5.

16. Ganz RA. A Modern Magnetic Implant for Gastroesophageal Reflux Disease. Clin Gastroenterol Hepatol 2017;15:1326-1337.

17. Bonavina L, DeMeester T, Fockens $P$, et al. Laparoscopic sphincter augmentation device eliminates reflux symptoms and normalizes esophageal acid exposure: one- and 2-year results of a feasibility trial. Ann Surg 2010;252:857-62.

18. Lipham JC, DeMeester TR, Ganz RA, et al. The LINX(R) reflux management system: confirmed safety and efficacy now at 4 years. Surg Endosc 2012;26:2944-9.

19. Ayazi S, Zheng P, Zaidi AH, et al. Magnetic Sphincter Augmentation and Postoperative Dysphagia: Characterization, Clinical Risk Factors, and Management. J Gastrointest Surg 2019.

20. Velanovich V. The development of the GERD-HRQL symptom severity instrument. Dis Esophagus 2007;20:130-4.

21. Velanovich $\mathrm{V}$, Karmy-Jones R. Measuring gastroesophageal reflux disease: relationship between the Health-Related Quality of Life score and physiologic parameters. Am Surg 1998;64:649-53.

22. Velanovich V, Vallance SR, Gusz JR, et al. Quality of life scale for gastroesophageal reflux disease. J Am Coll Surg 1996;183:217-24.

23. Aiolfi A, Asti E, Bernardi D, et al. Early results of magnetic sphincter augmentation versus fundoplication for gastroesophageal reflux disease: Systematic review and meta-analysis. Int J Surg 2018;52:82-88. 
24. Abdelmoaty WF, Dunst CM, Filicori F, et al. Combination of Surgical Technique and Bioresorbable Mesh Reinforcement of the Crural Repair Leads to Low Early Hernia Recurrence Rates with Laparoscopic Paraesophageal Hernia Repair. J Gastrointest Surg 2020;24:1477-1481.

25. Landreneau RJ, Del Pino M, Santos R. Management of paraesophageal hernias. Surg Clin North Am 2005;85:411-32.

26. Stylopoulos N, Gazelle GS, Rattner DW. Paraesophageal hernias: operation or observation? Ann Surg 2002;236:492-500; discussion 500-1.

27. DeMeester SR. Laparoscopic paraesophageal hernia repair: critical steps and adjunct techniques to minimize recurrence. Surg Laparosc Endosc Percutan Tech 2013;23:429-35.

28. Ayazi S, Chowdhury N, Zaidi AH, et al. Magnetic sphincter augmentation (MSA) in patients with hiatal hernia: clinical outcome and patterns of recurrence. Surg Endosc 2020;34:1835-1846.

29. Dreifuss NH, Schlottmann F, Molena D. Management of paraesophageal hernia review of clinical studies: timing to surgery, mesh use, fundoplication, gastropexy and other controversies. Dis Esophagus 2020;33.

30. Ayazi S, Grubic AD, Zheng P, et al. Measurement of outflow resistance imposed by magnetic sphincter augmentation: defining normal values and clinical implication. Surg Endosc 2020.

31. El Lakis MA, Kaplan SJ, Hubka M, et al. The Importance of Age on Short-Term Outcomes Associated With Repair of Giant Paraesophageal Hernias. Ann Thorac Surg 2017;103:1700-1709.

32. Schwameis K, Ayazi S, Zheng P, et al. Efficacy of Magnetic Sphincter Augmentation across the Spectrum of GERD Disease Severity. J Am Coll Surg 2020.

33. Bradley DD, Louie BE, Farivar AS, et al. Assessment and reduction of diaphragmatic tension during hiatal hernia repair. Surg Endosc 2015;29:796-804.

34. Zehetner J, DeMeester SR, Ayazi S, et al. Laparoscopic wedge fundectomy for collis gastroplasty creation in patients with a foreshortened esophagus. Ann Surg 2014;260:1030-3.

35. Antonoff MB, D'Cunha J, Andrade RS, et al. Giant paraesophageal hernia repair: technical pearls. J Thorac Cardiovasc Surg 2012;144:S67-70.

36. Swanstrom LL, Marcus DR, Galloway GQ. Laparoscopic Collis gastroplasty is the treatment of choice for the shortened esophagus. Am J Surg 1996;171:477-81.

37. Granderath FA, Carlson MA, Champion JK, et al. Prosthetic closure of the esophageal hiatus in large hiatal hernia repair and laparoscopic antireflux surgery. Surg Endosc 2006;20:367-79.

38. Oelschlager BK, Pellegrini CA, Hunter J, et al. Biologic prosthesis reduces recurrence after laparoscopic paraesophageal hernia repair: a multicenter, prospective, randomized trial. Ann Surg 2006;244:481-90.

39. Greene CL, DeMeester SR, Zehetner J, et al. Diaphragmatic relaxing incisions during laparoscopic paraesophageal hernia repair. Surg Endosc 2013;27:4532-8.

\section{Figures}




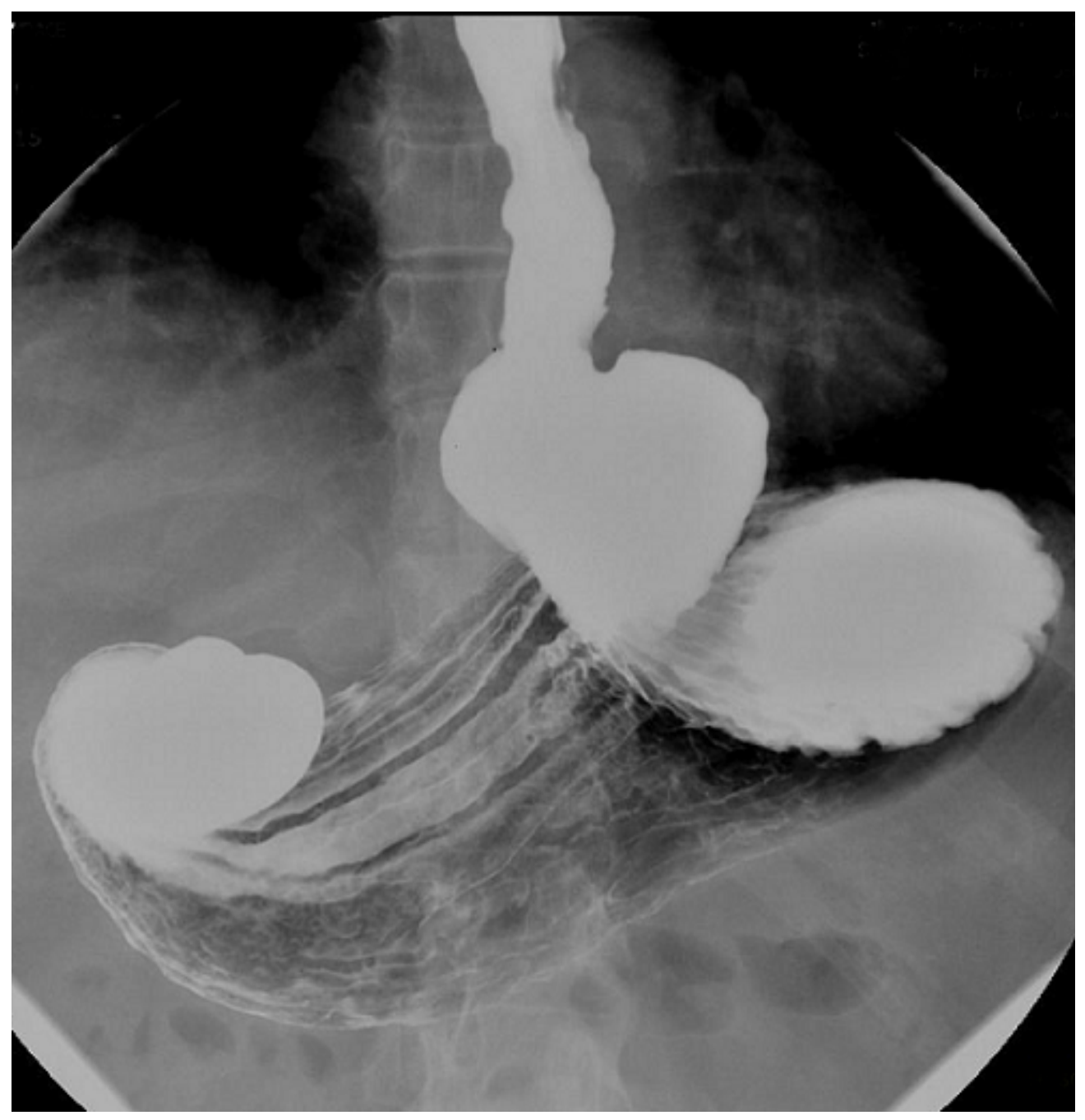

\section{Figure 1}

Preoperative esophagram in a patient with a paraesophageal hernia 


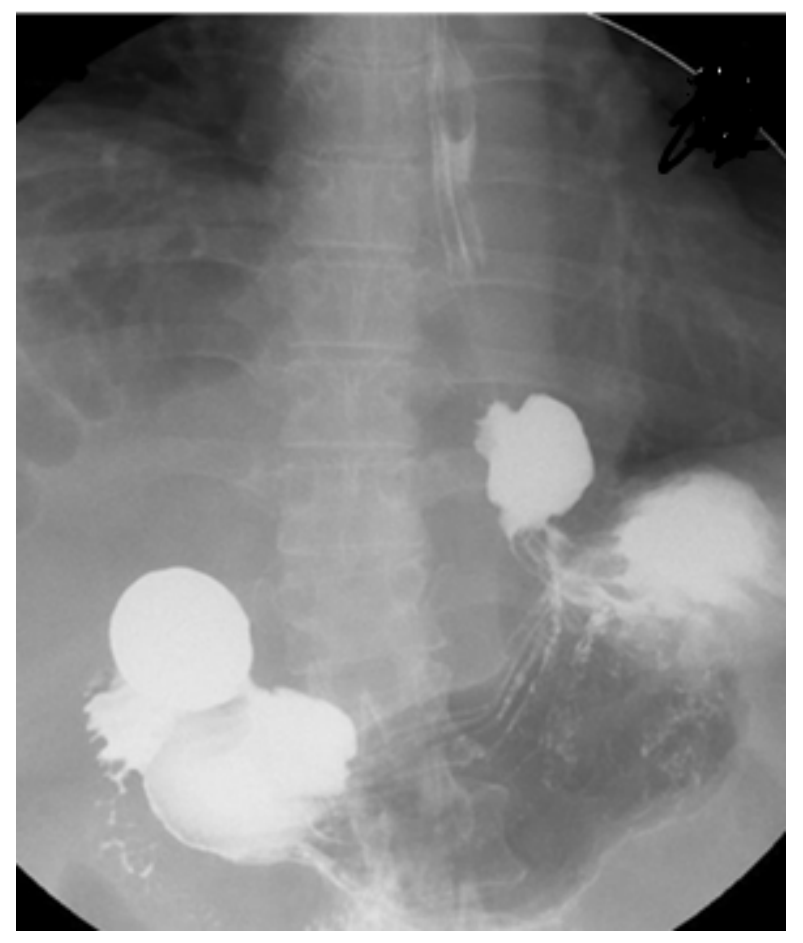

Figure 2

Preoperative esophagram in a patient with a large sliding hiatal hernia 


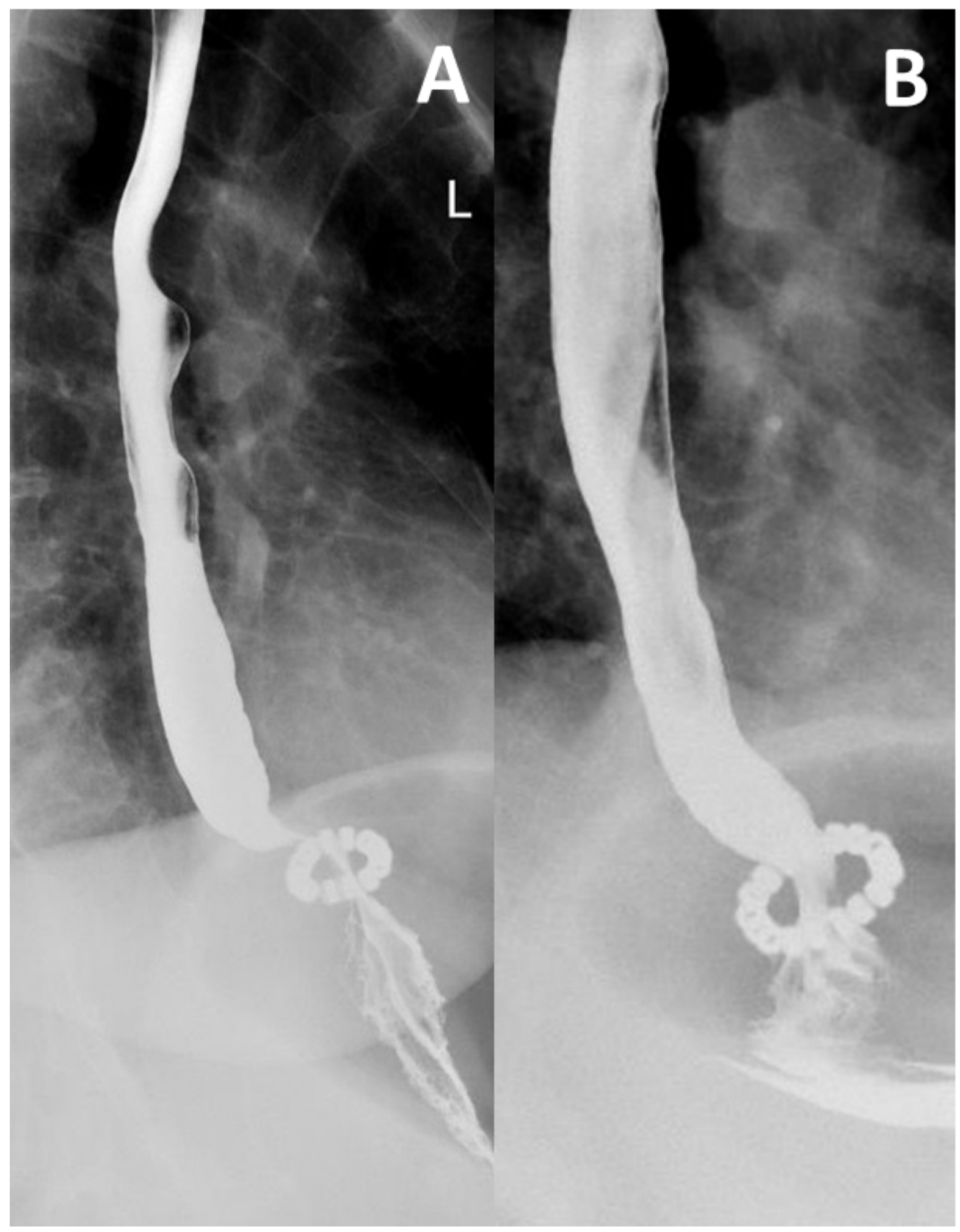

Figure 3

Postoperative esophagram after hernia repair and magnetic sphincter augmentation in a patient with (A) paraesophageal hernia and (B) large sliding hiatal hernia 
small asymptomatic

$18 \%$

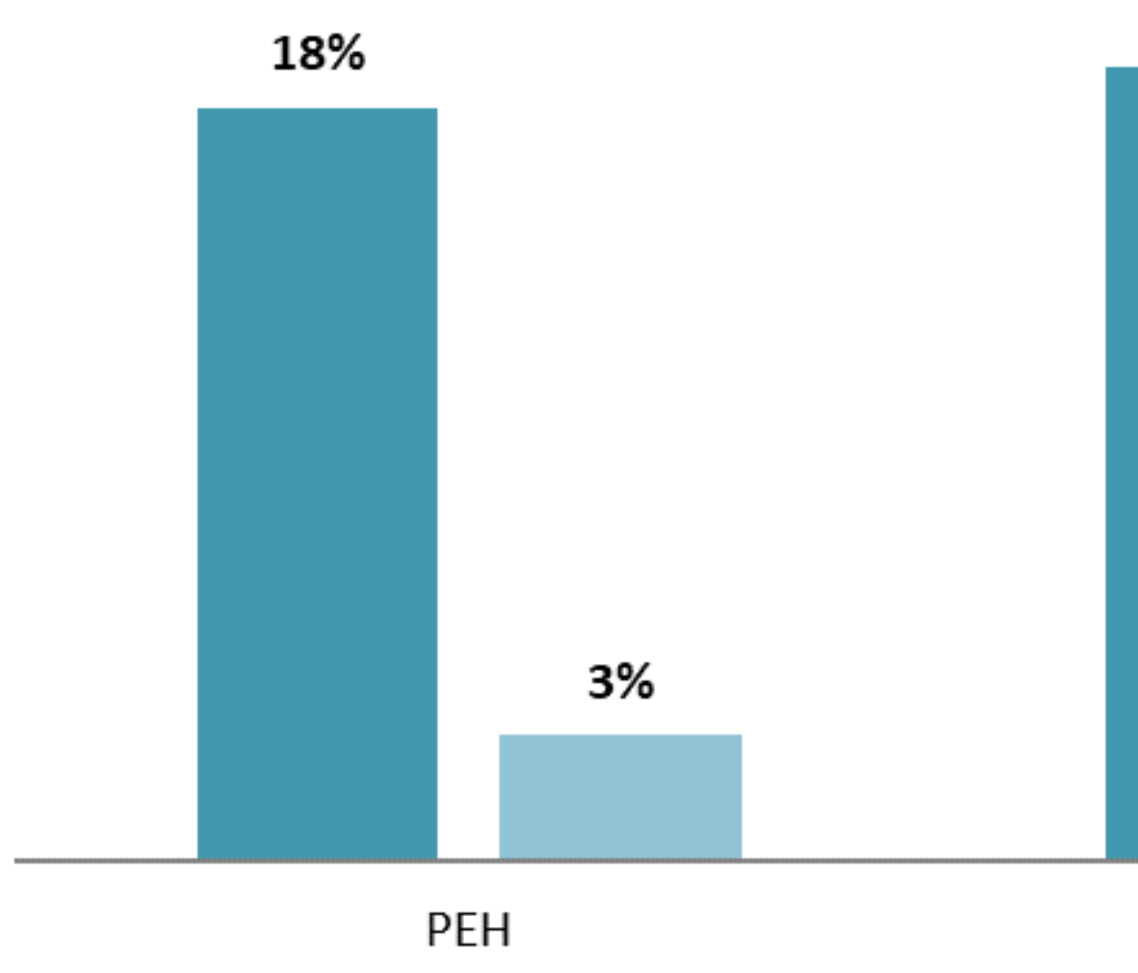

symptomatic, revision surgery

$19 \%$

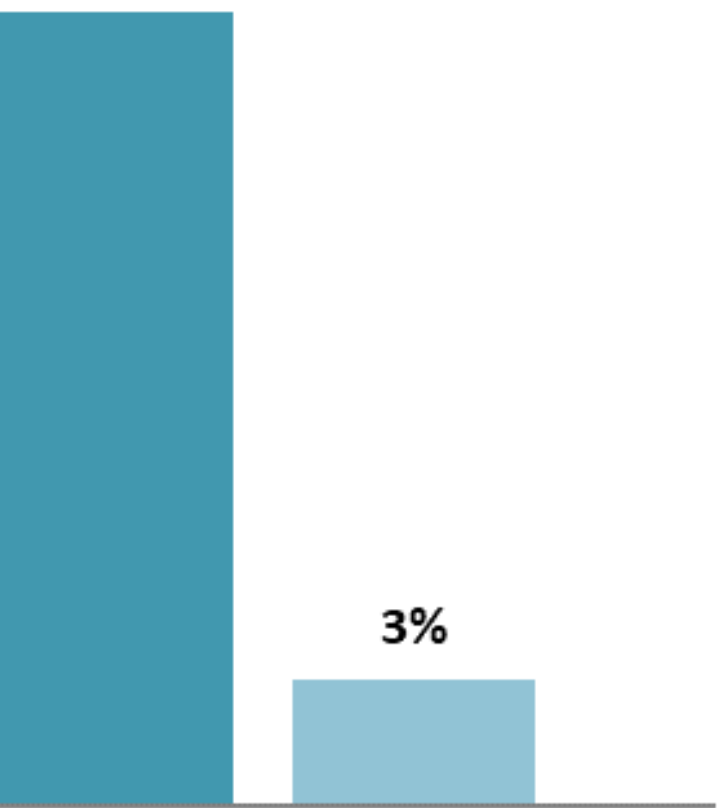

$\mathrm{LHH}$

\section{Figure 4}

Rates of asymptomatic small hernia recurrence and symptomatic recurrence requiring surgical intervention in patients with paraesophageal hernia (PEH) and large sliding hiatal hernia (LHH) 